\title{
Identidad ambiental, actitud y comportamiento de conservación de agua en una comunidad alto-andina del Perú
}

\section{Environmental identity, attitude and behavior of water conservation in a high-Andean community of Peru}

Daniel, Carbajal ${ }^{1,2,3}$; Ana, Cornejo ${ }^{1,2}$; Andy, Alvarado ${ }^{1,2}$; Liz, Salinas ${ }^{1,2}$; Rocío, León ${ }^{1,2}$ y Fredy, Monge ${ }^{1,2}$.

${ }^{1}$ Centro de Investigación Ambiente, Comportamiento y Sociedad (CIACOMS)

${ }^{2}$ Universidad Nacional de San Antonio Abad del Cusco-Perú

${ }^{3}$ Miniserio de Educación

Autorde correspondencia.Correo electrónico:goethedan@gmail.com

\begin{abstract}
Resumen
Se investigó la identidad ambiental, actitud y comportamiento de conservación de agua en habitantes de una comunidad altoandina de Perú $(\mathrm{N}=211)$ con el objetivo de determinar las relaciones entre ellas. se aplicó la escala de identidad ambiental y el cuestionario de actitudes y comportamientos de conservación de agua y se procedió a medir sus correlaciones. Se encontró una relación directa entre identidad ambiental, actitud y comportamiento de conservación de agua. La correlación más significativa se da entre identidad y comportamiento, en tanto las correlaciones entre identidad y actitud, actitud y comportamiento, son moderadas. Por otro lado, las dimensiones de la identidad ambiental muestran correlaciones significativas positivas, con la actitud, en tanto el comportamiento presenta correlaciones significativas con la autoidentificación, las emociones positivas y la relevancia de la identidad y la ideología. Nuestros hallazgos, indican que la identidad ambiental mantiene una relación sobre la actitud de conservación del agua. Se observó que, la identidad y/o conexión con la naturaleza motiva a proteger y actuar en favor de ella. Finalmente se discuten las implicancias del comportamiento proambiental sobre la identidad ambiental y la actitud para la conservación del agua.
\end{abstract}

Palabras clave: identidad ambiental, actitud, comportamiento de conservación de agua. 


\begin{abstract}
We investigated the environmental identity, attitude and behaviour of water conservation in inhabitants of a high Andean community in Peru $(\mathrm{N}=211)$ in order to determine the relationships between them. We applied the environmental identity scale and the questionnaire on water conservation attitudes and behaviour and proceeded to measure their correlations. A direct relationship was found between environmental identity, attitude and water conservation behaviour. The most significant correlation is between identity and behaviour, while the correlations between identity and attitude, attitude and behaviour, are moderate. On the other hand, the dimensions of environmental identity show significant positive correlations with attitude, while behaviour shows significant correlations with self-identification, positive emotions and the relevance of identity and ideology. Our findings indicate that environmental identity has a relationship with water conservation attitude. It was observed that, identity and/or connection with nature motivates people to protect and act in favour of it. Finally, the implications of pro-environmental behaviour on environmental identity and attitude to water conservation are discussed.
\end{abstract}

Key words: environmental identity, water conservation behavior, attitudes.

\title{
Introducción
}

La psicología ambiental del siglo XXI tiene el desafío de responder a la crisis climática. Entre las problemáticas más importantes se encuentra el uso sostenible del agua. Las ciencias biofísicas han concentrado esfuerzos en desarrollar tecnologías para su uso eficiente, sin embargo, las investigaciones de corte social y comportamental no han estado ausentes, aunque, no han tenido un rol importante debido a la tendencia a percibir la crisis ambiental como un problema para los físicos o geólogos y no tanto así para los científicos sociales o del comportamiento.

La presente investigación estudió el comportamiento y actitudes de conservación de agua, y su relación con la identidad ambiental; es decir, si la conexión con la naturaleza, que influye en nuestra manera de ocupar un territorio y utilizar recursos como el agua tienen relación con la conservación del agua; considerando que la identificación con la naturaleza contribuye a un cuidado de la misma por la significancia atribuida (Clayton, 2003).Además, informes como el Acuerdo de Paris, que suscribió el Perú, declara dentro de sus compromisos la "conservación de la disponibilidad hídrica para uso poblacional, ante peligros antrópicos asociados al cambio climático" (ANA, 2017). Asimismo, urge una "investigación científica y aplicada, el desarrollo de capacidades y la difusión del conocimiento para la adaptación al cambio climático" (MINAGRI, 2015), en tal sentido, la investigación se enmarca en abordar conductas sustentables enfocados en la conservación del agua desde un abordaje 
psicológico, social y ambiental. Por otro lado, existe la emergencia de una nueva cultura del agua que permita entender la gestión integral de recursos hídricos desde un enfoque no solohidrológico sino tambiénhumano, subrayando la naturalezacomportamental de la conservación del agua.

En la literatura científica se cuenta con estudios acerca de la identidad ambiental y los comportamientos proambientales en general (Clayton y Opotow, 2003), sin embargo son escasos los trabajos que han contemplado la relación directa entre las actitudes y los comportamientos de conservación de agua (Carbajal, 2019). Whitmarsh y O'Neill (2010) sostienen que la autoidentidad ambiental en contraste con la conducta planificada predice de manera más segura las conductas proambientales. Por su parte Werff, Steg y Keizer (2013) afirman que las acciones proambientales guardan relación con la autoidentidad ambiental por medio de las obligaciones o del deber moral. Para Olivos, Talayero, Talayero, Aragonés y Moyano-Díaz (2014) las dimensiones del comportamiento proambiental se asocian a la identidad ambiental y a la conectividad con la naturaleza.

El ahorro de agua se explicó a partir de las normas subjetivas, la percepción de control y la información sobre conservación de agua, mientras que la actitud hacia este recurso no necesariamente se manifiesta de forma similar, (Perren y Yang, 2015), esto mismo en términos de Dolnicar y Hurlimann (2010) quiere decir que las actitudes de conservación de agua no garantizan en efecto que la persona conserve el agua; es más un público informado se inclina a la adopción de conductas orientadas al ahorro de agua.

"Lograr una mayor conservación del agua es fundamental para garantizar la gestión sostenible de los recursos hídricos y es especialmente importante a la luz de las cambiantes condiciones climáticas" (Dolnicar y cols., 2012), el presente estudio tiene como objetivo determinar de qué manera se relaciona la identidad ambiental con la actitud y el comportamiento deconservación deaguapara asíconocersi elfortalecimiento delaidentidad ambiental contribuye a mejorar las actitudes y los comportamientos de conservación de agua.

\section{Método}

La conforman habitantes de una comunidad campesina de los andes del Perú nombre de la comunidad, donde un $50.7 \%(\mathrm{n}=107)$ eran mujeres y el $49.3 \%$ varones $(n=104)$. El 39.80\% lograron secundaria completa o incompleta y un 37.40\% educación primaria. Los entrevistados son en su mayoría agricultores y criadores de camélidos. Indicar procedimientos de ética para el estudio, básicamente describir el consentimiento de informado. Se aplicaron la escala de identidad ambiental desarrollada por Clayton (2003), agrupa 24 ítems y tiene siete opciones de respuesta (nada cierto, ni verdadero ni falso, completamente falso).

Evalúa la relevancia de la identidad, autoidentificación, ideología y emociones positivas. Su alfa de Cronbach es de 0.79 . El cuestionario de actitudes y comportamientos de conservación de agua formulada por Dolnicar y Hurlimann (2010) se divide en dos segmentos, el cuestionario de actitudes conformado por 15 ítems, el de comportamiento 
tiene 16 ítems. Los valores del alfa de Cronbach son de 0.60 y 0.62 respectivamente. El cuestionario toma en cuenta una escala "Likert" que va desde muy alto y alto, pasando por regular y llega hasta bajo y muy bajo y tiene como opciones de respuesta para las actitudes "de acuerdo"y en "desacuerdo", y para el comportamiento las opciones "si"y "no".

Asimismo, se aplicó una encuesta sociodemográfica para conocer la edad, grado de instrucción y sexo. Se contó con la colaboración de siete encuestadores para la recolección de datos, los mismos que fueron entrenados en la aplicación de los instrumentos. Las entrevistas se efectuaron en castellano y quechua en diferentes visitas a la comunidad. Además, se aplicó un consentimiento informado escrito a todos los encuestados. Se realizaron tablas de contingencia para analizar las frecuencias, medias, desviaciones estándar y porcentajes. Se empleó la $\mathrm{r}$ de Pearson para medir el grado de correlación entre las variables, donde 0 indica ausencia de correlación y 1 correlación perfecta. Se evaluó la significancia y dirección de las correlaciones.

\section{Resultados}

El análisis descriptivo encontró que los niveles de identidad ambiental se encuentran en un nivel bajo y muy bajo (41.8\%), siendo similares a los niveles alto y muy alto (36.5\%). En la mayoría de casos la identidad ambiental presenta un grado medio con un $22.7 \%$ y la actitud tiende hacia un nivel muy bajo y bajo de conservación de agua (43.1\%). El nivel de actitud de conservación del agua alcanza un $25 \%$ con un nivel regular. El comportamiento de conservación de agua se encuentra en un grado medio. Por otro lado, hay una tendencia al nivel alto y muy alto de comportamiento de conservación de agua (32.2\%), en seguida se tiene a los niveles bajo y muy bajo que sumados llegan a un 30.3\%. Estos resultados se pueden apreciar en la Tabla 1.

\section{Tabla 1}

Frecuencias descriptivas de identidad ambiental, actitud y comportamiento de conservación de agua

\begin{tabular}{lcccccc}
\hline & \multicolumn{2}{c}{ Identidad ambiental } & \multicolumn{2}{c}{ Actitud } & \multicolumn{2}{c}{ Comportamiento } \\
\hline & $\mathrm{f}$ & $\%$ & $\mathrm{f}$ & $\%$ & $\mathrm{f}$ & $\%$ \\
\cline { 2 - 7 } Muy Bajo & 45 & $21.30 \%$ & 48 & $22.70 \%$ & 57 & $27.00 \%$ \\
Bajo & 41 & $19.50 \%$ & 43 & $20.40 \%$ & 28 & $13.30 \%$ \\
Medio & 48 & $22.70 \%$ & 53 & $25.10 \%$ & 58 & $27.50 \%$ \\
Alto & 35 & $16.60 \%$ & 30 & $14.20 \%$ & 34 & $16.10 \%$ \\
Muy alto & 42 & $19.90 \%$ & 37 & $17.50 \%$ & 34 & $16.10 \%$ \\
\hline Total & 211 & $100 \%$ & 211 & $100 \%$ & 211 & $100 \%$
\end{tabular}


El análisis comparativo indica que la identidad ambiental es mayor en las mujeres que en los varones, ya que alcanzan un puntaje de $44 \%$ con niveles altos y muy altos, en tanto los varones puntúan muy bajo y bajo (53\%). Existe una mayor identidad ambiental entre los adultos de 54 a 67 años. Los participantes de 18 a 26 años obtuvieron una identidadmuybaja obaja.Laidentidadambiental novaríaenfuncióndelgradodeinstrucción.

\section{Tabla 2}

Análisis comparativo de variables sociodemográficas respecto a identidad ambiental

Identidad ambiental

\begin{tabular}{lccccccc}
\hline & Muestra total=211 & Muy Bajo & Bajo & Medio & \multicolumn{2}{c}{ Alto } & Muy alto \\
\cline { 2 - 7 } Género & $\mathrm{N}$ & $\mathrm{N}$ & $\mathrm{N}$ & $\mathrm{N}$ & $\mathrm{N}$ & $\mathrm{N}$ \\
\hline Femenino & 107 & 15 & 15 & 29 & 21 & 27 \\
Masculino & 104 & 30 & 26 & 19 & 14 & 15 \\
\hline Edad & & & & & & \\
\hline 18 a 26 años & 26 & 9 & 10 & 1 & 2 & 4 \\
27 a 40 años & 64 & 16 & 13 & 12 & 9 & 14 \\
41 a 53 años & 39 & 6 & 5 & 11 & 8 & 9 \\
54 a 67 años & 48 & 6 & 6 & 15 & 10 & 11 \\
67 a más años & 34 & 8 & 7 & 9 & 6 & 4 \\
\hline Grado de instrucción & & & & & & \\
\hline Primaria completa & 66 & 8 & 12 & 15 & 12 & 19 \\
Primaria incompleta & 13 & 6 & 1 & 2 & 1 & 3 \\
Secundaria completa & 31 & 7 & 8 & 7 & 5 & 4 \\
Secundaria incompleta & 53 & 11 & 6 & 17 & 8 & 11 \\
Técnico superior completa & 14 & 3 & 7 & 1 & 2 & 1 \\
Técnico superior incompleta & 17 & 3 & 6 & 3 & 3 & 2 \\
Superior completa & 1 & 0 & 0 & 0 & 1 & 0 \\
Superior incompleta & 16 & 7 & 1 & 3 & 3 & 2 \\
\hline
\end{tabular}

La actitud de conservación de agua obtiene un nivel alto y muy alto en las mujeres (33\%), los varones presentan puntajes más altos en los niveles bajo y muy bajo (46\%). En ambos sexos se tiene un nivel medio de identidad ambiental. Sin embargo, los participantes de entre 54 a 67 años de edad presentan niveles altos y muy altos. En tanto los comuneros de entre 27 a 40 años tienden a niveles bajo y muy bajo de actitud de conservación de agua. En promedio los participantes tienden a puntuar en el nivel medio. La actitud de conservación de agua en pobladores con primaria completa tiende a niveles muy alto y medio. 


\section{Tabla 3}

Análisis comparativo de variables sociodemográficas respecto a actitud de conservación de agua

Actitud

\begin{tabular}{lcccccc}
\hline \multirow{2}{*}{ Género } & Muestra total=211 & Muy Bajo & Bajo & Medio & Alto & Muy alto \\
\cline { 2 - 7 } & $\mathrm{N}$ & $\mathrm{N}$ & $\mathrm{N}$ & $\mathrm{N}$ & $\mathrm{N}$ & $\mathrm{N}$ \\
\hline Femenino & 107 & 24 & 19 & 28 & 15 & 21 \\
Masculino & 104 & 24 & 24 & 25 & 15 & 16 \\
\hline Edad & & & & & & \\
\hline 18 a 26 años & 26 & 7 & 5 & 9 & 1 & 4 \\
27 a 40 años & 64 & 16 & 17 & 17 & 5 & 9 \\
41 a 53 años & 39 & 10 & 11 & 7 & 5 & 6 \\
54 a 67 años & 48 & 9 & 6 & 11 & 11 & 11 \\
67 a más años & 34 & 6 & 4 & 9 & 8 & 7 \\
\hline Grado de instrucción & & & & & & \\
\hline Primaria completa & 66 & 10 & 12 & 19 & 9 & 16 \\
Primaria incompleta & 13 & 5 & 3 & 3 & 2 & 0 \\
Secundaria completa & 31 & 11 & 2 & 9 & 3 & 6 \\
Secundaria incompleta & 53 & 3 & 1 & 7 & 2 & 1 \\
Técnico superior incompleta & 17 & 4 & 6 & 1 & 2 & 4 \\
Superior completa & 1 & 0 & 1 & 0 & 0 & 0 \\
Superior incompleta & 16 & 4 & 5 & 3 & 2 & 2 \\
\hline
\end{tabular}

El comportamiento de conservación de agua es de mayor nivel en las mujeres con puntajes alto y muy alto (42\%), los varones por su parte tienden a un comportamiento con niveles "muy bajo" y "bajo" (49\%). Siendo así, las mujeres muestran mayor comportamiento de conservación de agua respecto a los varones. Los comuneros que presentan una edad de entre 54 a 67 años alcanzan niveles altos y muy altos de comportamiento de conservación de agua.

\section{Tabla 4}

Análisis comparativo de variables sociodemográficas respecto a comportamiento de conservación de agua 
Comportamiento

\begin{tabular}{lcccccc}
\hline & Muestra total=211 & Muy Bajo & Bajo & Medio & Alto & Muy alto \\
\cline { 2 - 7 } Género & $\mathrm{N}$ & $\mathrm{N}$ & $\mathrm{N}$ & $\mathrm{N}$ & $\mathrm{N}$ & $\mathrm{N}$ \\
\hline Femenino & 107 & 25 & 9 & 28 & 20 & 25 \\
Masculino & 104 & 32 & 19 & 30 & 14 & 9 \\
\hline Edad & & & & & & \\
\hline 18 a 26 años & 26 & 11 & 8 & 5 & 2 & 0 \\
27 a 40 años & 64 & 21 & 8 & 17 & 9 & 9 \\
41 a 53 años & 39 & 8 & 4 & 12 & 6 & 9 \\
54 a 67 años & 48 & 6 & 7 & 13 & 13 & 9 \\
67 a más años & 34 & 11 & 1 & 11 & 4 & 7 \\
\hline Grado de instrucción & & & & & & \\
\hline Primaria completa & 66 & 15 & 6 & 23 & 7 & 15 \\
Primaria incompleta & 13 & 6 & 1 & 2 & 2 & 2 \\
Secundaria completa & 31 & 10 & 4 & 7 & 5 & 5 \\
Secundaria incompleta & 53 & 11 & 7 & 14 & 11 & 10 \\
Técnico superior completa & 14 & 4 & 1 & 4 & 4 & 1 \\
Técnico superior incompleta & 17 & 4 & 4 & 4 & 4 & 1 \\
Superior completa & 1 & 0 & 1 & 0 & 0 & 0 \\
Superior incompleta & 16 & 7 & 4 & 4 & 1 & 0 \\
\hline
\end{tabular}

En general existe una tendencia a un nivel medio y muy bajo en pobladores de 27 a 40 años. Por todo esto, el comportamiento de conservación de agua varía según el grupo etario. Por último, no existe una variación significativa al comparar el comportamiento de conservación de agua según el grado de instrucción. La tabla 5, nos muestra las correlaciones entre las variables de estudio, se observa la correlación de .19, entre la identidad ambiental y la actitud de conservación de agua. Por otro lado, se tiene una relación directa significativa entre la identidad ambiental y el comportamiento de conservación de agua .46, en los pobladores de la Comunidad Campesina de Chillca.

Finalmente, el coeficiente de correlación de Pearson es de .21, respecto a la relación entre actitud y el comportamiento de conservación de agua. Del mismo modo se observa un coeficiente de correlación de .14, entre la relevancia de la identidad y la actitud de conservación de agua. Se observa una relación directa débil entre la autoidentificación y la actitud de conservación de agua .15. Se puede observar una relación directa entre la actitud de conservación de agua y la ideología .19. La relación entre las emociones positivas y la actitud de conservación de agua presenta un coeficiente correlación .06 , lo cual representa una correlación directa muy débil.

La relación entre la relevancia de la identidad y el comportamiento de conservación de agua en los pobladores de la Comunidad de Chillca presente un coeficiente de correlación de .34. La correlación $\mathrm{r}$ de Pearson muestra un coeficiente de .27, entre la autoidentificación y el comportamiento de conservación de agua en los pobladores de la Comunidad de Chillca. El coeficiente de correlación de Pearson .42 representa una relación directa significativa entre el comportamiento de conservación de agua y la ideología. La relación entre las emociones positivas y el comportamiento de conservación de agua fue directa media debido al coeficiente 35 . 
En resumen, se encontró una correlación directa mayor en cuanto a la identidad ambiental y el comportamiento de conservación de agua, seguido de actitud y comportamiento y finalmente identidad ambiental y actitud, lo que permite inferir que en la conservacióndelaguainfluyenfactorespsicológicos, socialesyculturales, loquenosconducea completar las intervenciones en el uso eficiente de este recurso vital a través de estudios comportamentales.

\section{Tabla 5}

Correlaciones de las variables de estudio

\begin{tabular}{llll}
\hline Variables & 1 & 2 & 3 \\
\hline 1. Identidad ambiental & 1 & & \\
2. Actitud & $.19^{* *}$ & .1 & \\
3. Comportamiento & $.46^{* *}$ & $.21^{* *}$ & 1 \\
\hline
\end{tabular}

Nota: $*$ indica $\mathrm{p}<.05 . * *$ indica $\mathrm{p}<.01$.

La relación entre identidad ambiental, actitud y comportamiento de conservación de agua es directa.

\section{Discusión}

El presente estudio encontró una relación directa entre identidad ambiental, actitud y comportamiento de conservación de agua (Tabla 5). La correlación más significativa radica en la relación identidad ambiental y comportamiento, en tanto la relación entre la identidad y actitud, actitud y comportamiento muestran correlaciones débiles, por lo que el comportamiento de conservación de agua no deriva necesariamente de una actitud favorable hacia su conservación. Considerando que la identidad ambiental resulta influyendo significativamente en el comportamiento de conservación de agua, es importante incorporarla como factor adicional en la teoría de la conducta planificada, como sugiere Carfora, Caso, Sparks y Conner (2017). Esto permitirá una mayor comprensión de los factores que influyen en el comportamiento de conservación de agua.

Los datos encontrados indican que la identidad ambiental mantiene una relación sobre la actitud de conservación del agua, como sostuvo Clayton (2003), al afirmar que la identidad ambiental tiene una relación positiva con las actitudes proambientales. Se halló una correlación entre identidad ambiental y el comportamiento de conservación de agua tal como Whitmarsh y O'Neill (2010) expusieron en sus investigaciones donde la autoidentidad ambiental predecía el comportamiento de conservación de agua. La identidad y/o conexión con la naturaleza motiva a protegerla y actuar en favor de ella (Nigbur, Lyons y Uzzell, 2010; Whitburn, Linklater y Milfont, 2018; Schmitt, Mackay, Droogendyk y Payne, 2018). Para Olivos y cols. (2014) el comportamiento proambiental tiene relación con la identidad ambiental, es decir que 
las personas que viven en entornos naturales, que consideran la naturaleza como parte de su identidad y manifiestan estar de acuerdo con cuidar el medioambiente, se inclinan más a conservar la naturaleza y elementos a decir del agua (Clayton, 2012a).

Hallazgos similares fueron reportados por investigadores chinos, estos evidenciaron que la sensación de estar conectado con la naturaleza induce a una menor probabilidad de participar en comportamientos perjudiciales al medioambiente (Liu, Geng, Ye y Zhou, 2019), lo que explicaría la asociación entre identidad ambiental y el comportamiento de conservación de agua. Un estudio en Inglaterra aseveró que las personas con pobre conexión con la naturaleza tenían una menor probabilidad a realizar comportamientos de conservación (Hughes, Richardson y Lumber, 2018). La identidad ambiental conduce al deber moral de ser amigables con la naturaleza (van der Werff y cols., 2013); asimismo, las personas que presentan una fuerte identificación con la naturaleza, tienen valores biosféricos y registran conductas ambientales pasadas (van der Werff, Steg, y Keizer, 2014).

El estudio meta analítico de los determinantes psicosociales de la conducta proambiental encontró que la actitud alcanzó una correlación similar al presente estudio (Bamberg y Möser, 2007). El estudio de Moore y cols. (1994) es similar a la investigación ya que la actitud estuvo asociada al comportamiento de ahorro de agua, su influencia era indirecta por medio de la intención conductual. Esto implica que las personas que están predispuestas a ahorrar o tener un cuidado al usar agua se sienten más comprometidas para ahorrar y, por tanto, su intención para conservar el agua es mayor (Nigbur y cols., 2010).

Por otro lado, estos hallazgos no se corresponden con los de Perren y Yang (2015) donde la actitud no predijo el ahorro de agua. La actitud influye, mas no constituye un principal predictor del comportamiento proambiental como la conservación del agua, en otros términos, la actitud a favor del ambiente no se asegura una acción a favor de la naturaleza (Dolnicar y Hurlimann, 2010); lo anterior también puede explicarse desde la Teoría de la Disonancia Cognitiva (Festinger, 1975) donde las actitudes proambientales no se convierten automáticamente en conductas de conservación. En resumen, este hallazgo refuerza la teoría de la conducta planificada, en la que el papel de las actitudes sobre los comportamientos juega un rol relevante más no determinante (Ajzen, 1991).

Asimismo, el presente estudio encontró una correlación positiva entre las emociones y el comportamiento de conservación de agua. Estos resultados se asemejan a los hallazgos de Peçanha, Veloso, Silva, Milfont y Romariz (2016) y Vining y Ebreo (2002) donde las emociones positivas conducían a una motivación al comportamiento de conservación ambientaly una afinidad con la naturaleza(Vining y Ebreo, 2002). De acuerdo a Hinds y Sparks (2008), quienes demuestran que la conexión emocionalo afectiva con la naturaleza induce a las personas a comprometerse con la naturaleza. Por otro lado, el hecho de que los pobladores hayan vivido cerca de la naturaleza hace que presenten una mayor conexión afectiva positiva con la naturaleza y por ende tengan un comportamiento de conservación de agua más sólido (Hinds y Sparks, 2008). 
Tomando en cuenta que el estudio del comportamiento de conservación de agua se recogió a partir de las conductas de autoreporte, las mismas que pueden presentan sesgos al momento de comunicar sus conductas por el factor de deseabilidad social (Dolnicar y cols., 2012; Moore y cols., 1994), se requiere a futuro promover investigaciones que analicen la conservación del agua de permita la cuantificación, que podría ser el medidor de agua o una ficha de observación de los comportamientos de conservación de agua.

Las limitaciones del estudio se enmarcan en las conductas de autoreporte, donde el factor de deseabilidad social influye en la respuesta de los encuestados (Dolnicar y cols., 2012; Moore y cols., 1994). Para una medir la conservación del agua de manera más exacta se requiere indicadores métricos o ejecutar una ficha de observación de comportamientos. Los próximos estudios psicológicos deben seguir concentrandosé en ecosistemas de alta montaña vulnerables a los impactos del cambio climático, incidiendo en la respuesta humana y en la adaptación conductual de las comunidades campesinas a problemas como la desglaciación, que afectan la disponibilidad del agua.

Estos hallazgos revelan la importancia de la identidad ambiental para desarrollar o propiciar actitudes y comportamientos de conservación de agua, por medio de la educación ambiental dirigida a los ciudadanos. También se observa la necesidad de generar un plan de gestión integral del agua que considere aspectos o dimensiones comportamentales para una mejor conservación del agua.

\section{Agradecimientos}

Con sincera admiración al docente investigador y pionero de la Psicología Ambiental en los andes del Perú, al Dr. Fredy S. Monge Rodríguez por la constante motivación en la producción científica de este presente artículo de investigación. Sinceros agradecimientos a todos los integrantes del Centro de investigación Ambiente, Comportamiento y Sociedad por su cooperación en esta empresa académica al servicio de un desarrollo sostenible para las comunidades altoandinas del Perú profundo.

\section{Declaración de conflicto de intereses}

No existe algún interés más que el de llegar a nuestros lectores y contribuir a la investigación. 


\section{Referencias}

Ajzen, I. (1991). The theory of planned behavior. Orgnizational Behavior and Human Decision Processes, 50, 179-211. https://doi.org/10.1016/0749-5978(91)90020-T

Alfaro, J. (2008). Conflictos, gestión del agua y cambio climático (Primera ed). Lima: Soluciones Prácticas-ITDG.

Bamberg, S. y Möser, G. (2007). Twenty years after Hines, Hungerford, and Tomera: A new meta-analysis of psycho-social determinants of pro-environmental behaviour. Journal of Environmental Psychology, 27(1), 14-25. https://doi.org/10.1016/j. jenvp.2006.12.002

Carfora, V., Caso, D., Sparks, P. y Conner, M. (2017). Moderating effects of pro-environmental self-identity on pro-environmental intentions and behaviour: A multi-behaviour study. Journal of Environmental Psychology, 53, 92-99. https:// doi.org/10.1016/j.jenvp.2017.07.001

Clayton, S. (2003). Environmental identity: A conceptual and an operational definition. En: S. Clayton y S. Opotow (Eds.), Identity and the Natural Environment: The Psychological Significance of Nature (pp. 45-65). Cambridge, MA: MIT Press.

Clayton, S. (2012a). Environment and Identity. En: S. Clayton (Ed.), The Oxford Handbook of Environmental and Conservation Psychology (pp. 164-180). New York: Oxford University Press.

Clayton, S., y Opotow, S. (2003). Identity and the Natural Environment. The Psychological Significance of Nature. Boston: MIT Press.

Corral, V. (2003). Determinantes psicológicos e situacionais do comportamento de conservação de água: um modelo estrutural. Estudos de Psicologia (Natal), 8(2), 245-252. https://doi.org/10.1590/S1413-294X2003000200006

Dolnicar, S. y Hurlimann, A. (2010). Australians' water conservation behaviours and attitudes. Australian Journal of Water Resources, 14(1), 43-53. https://doi.org/10 $.1080 / 13241583.2010 .11465373$

Dolnicar, S., Hurlimann, A. y Grün, B. (2012). Water conservation behavior in Australia. Journal of Environmental Management, 105, 44-52. https://doi.or $\mathrm{g} / 10.1016 / \mathrm{j} . j e n v m a n .2012 .03 .042$

Festinger, L. (1975). A theory of cognitive dissonance. Palo Alto: Stanford University Press.

Gilvonio, A. (2017). Seguimos mirando rio arriba. Lima: CooperAcción. 
Güimac, M., Almendras, J. L., Quevedo, J. y Puerta, S. M. (2018). Guía del promotor de cultura del Agua. (J. L. Carrillo, Ed.) (Primera ed). Lima: Autoridad Nacional del Agua ANA).

Hinds, J. y Sparks, P. (2008). Engaging with the natural environment: The role of affective connection and identity. Journal of Environmental Psychology, 28(2), 109-120. https://doi.org/10.1016/j.jenvp.2007.11.001

Hughes, J., Richardson, R. y Lumber, M. (2018). Evaluating connection to nature and the relationship with conservation behaviour in children. Journal for Nature Conservation, 45, 11-19. https://doi.org/10.1016/j.jnc.2018.07.004

Liu, T., Geng, L., Ye, L. y Zhou, K. (2019). "Mother Nature" enhances connectedness to nature and pro-environmental behavior. Journal of Environmental Psychology, 61(163), 37-45. Recuperado de: https://www. sciecedirect.com/science/article/pii/S0272494418302652?dgcid=rss_sd_all

Moore, S., Watson, R. y Murphy, M. (1994). A longitudinal study of domestic water conservation behavior. Population and Environment, 16(2), 175-189.

Nigbur, D., Lyons, E. y Uzzell, D. (2010). Attitudes, norms, identity and environmental behaviour: Using an expanded theory of planned behaviour to predict participation in a kerbside recycling programme. British Journal of Social Psychology, 49(2), 259-284. https://doi.org/10.1348/014466609X449395

Olivos, P., Talayero, F., Talayero, F.,Aragonés, J. I., y Moyano-Díaz, E. (2014). Dimensiones del Comportamiento Proambiental y su Relación con la Conectividad e Identidad Ambientales. Comportamento Pró-Ambiental e Sustentabilidade, 45(3), 369-376.

Ore, M., del Castillo, L., van Orsel, S. y Vos, J. (2009). El agua, ante nuevos desafios. Actores e iniciativas en Ecuador, Perú y Bolivia. (primera ed). Lima: IEP, OXFAM.

Peçanha, J. A., Veloso, V., Silva, G. H., Milfont, T. L., y Romariz, B. N. (2016). Emotions toward water consumption: Conservation and wastage. Revista Latinoamericana de Psicología, 48(2), 117-126. https://doi.org/10.1016/ j.rlp.2015.09.006

Perren, K. y Yang, L. (2015). Psychosocial and behavioural factors associated with intention to save water around the home: A Greek case study. Procedia Engineering, 119(1), 1447-1454. https://doi.org/10.1016/j.proeng.2015.08.1005

Schmitt, M. T., Mackay, C. M. L., Droogendyk, L. M. y Payne, D. (2018). What predicts environmental activism? The roles of identification with nature and politicized environmental identity. Journal of Environmental Psychology, 61(November 2018), 20-29. https://doi.org/10.1016/j.jenvp. 2018.11.003 
Schoolmeester, T., Johansen, K. S., Alfthan, B., Baker, E., Hesping, M. y Verbist, K. (2018). Atlas de Glaciares y Aguas Andinos. El impacto del retroceso de los glaciares sobre los recursos hídricos. (T. Schoolmeester y K. Verbist, Eds.). Arendal: UNESCO.

Sotomayor, M. A. y Choquevilca, W. (2011). La gente, la gestión del agua y el territorio(Primera ed). Cusco: Intercooperation, MINAGRI, COSUDE y Gobierno Regional Cusco.

Swim, J., Clayton, S., Doherty, T., Gifford, R., Howard, G., Reser, J., Weber, E. (2009). Psychology and Global Climate Change: Addressing a Multi-faceted Phenomenon and Set of Challenges (First Edit). Washington: American Psychological Association.

van der Werff, E., Steg, L., y Keizer, K. (2013). It is a moral issue: The relationship between environmental self-identity, obligation-based intrinsic motivation and pro-environmental behaviour. Global Environmental Change.

van der Werff, E., Steg, L., y Keizer, K. (2014). The influence of biospheric values and past behavior on environmental self-identity. Environment and Behavior, 46(5), 626-657. https://doi.org/10.1177/0013916512475209

Vining, J. y Ebreo, A. (2002). Emerging theoretical and methodological perspectives on conservation behavior. The New Handbook of Environmental Psychology, (August), 541-558. https://doi.org/10.1016/j.jenvp.2004.02.001 